\title{
The importance of HPV vaccination today
}

\author{
Reinhard Höpfl
}

Received: 7 January 2014 / Accepted: 11 February 2014 / Published online: 28 February 2014

(C) Springer-Verlag Wien 2014

In the 1930s, Richard Shope [1] unravelled a mystery about an animal hybrid between "jackrabbits" and "antalops"; these mythical "horned" cottontail rabbits called Jackalops were infected by an oncogenic viral agent. Half a century later, Nobel Prize laureate Harald zur Hausen [2] postulated that certain human papillomaviruses (HPV) cause cervical cancer by sexual transmission. Disappointingly, HPV-6 or -11 detected in genital warts were not found in cancer tissue. Instead, in 1985, weak hybridizing signals cross-reacting with HPV- 11 revealed the distantly related high-risk HPV types 16 and 18 to be present in cervical cancer. With support of his staff around Lutz Gissmann and in a worldwide academic concerted action, the mechanisms of HPV-induced carcinogenesis were subsequently elucidated. For example, HPV hampers apoptosis via deregulation of tumor suppressor functions of p53 and retinoblastoma protein, leading to immortalized (not yet malignant) cell lines vulnerable to second steps of transformation. Yet, most HPV infections resolve spontaneously. Thus, HPV testing without a wise concept of management for positive results could lead to unnecessary overtreatment.

As HPV does not grow in culture, only an athymic mouse xenograft model taught us that neutralizing (conferring protection from infection) antibodies strongly depend on antigen conformation [3]. In 1991, the production of 3D intact virus-like particles (VLP) in eukaryotic expression systems paved the way for vaccine development [4]. Unfortunately, the experimental progress in academic research about HPV vaccines was critically delayed due to adjudication of overlapping patent claims. After a 10-year "interference" Merck and

\footnotetext{
R. Höpfl, MD ( $\triangle)$

Department of Dermatology and Venereology,

Innsbruck Medical University,

Anichstraße 35, 6020 Innsbruck, Austria

e-mail: reinhard.hoepfl@i-med.ac.at
}

GlaxoSmithKline finally cross-licensed patents of all parties and launched two vaccines in the market with nearly identical price. The quadrivalent Gardasil ${ }^{\oplus}$ protects from HPV 6/11 (90\% of genital warts) and the high-risk types HPV16/18; the bivalent Cervarix ${ }^{\ominus}$ contains HPV 16/18 VLPs only. Targeting these two high-risk types could (with some cross-protection) potentially prevent $80 \%$ of invasive cervical cancers.

With cheaper and broader nonvalent vaccines ahead, neoplasms caused by HPV may face extinction [2]. Virtually every part of our skin is infected by mucosal or cutaneous HPV types. Aside short-term vaccination effects on nasty genital warts and on the huge number of atypical Pap smears, there will likely be a strong impact on additional cancers. Not only anogenital cancer-a particular problem in risk groups such as immunosuppressed or in men having sex with men-but also a relevant subset of head and neck squamous cell carcinomas is HPV-associated. Furthermore, there is the suspicion that other oncogenic HPV-types may also play a role in initiating UV-driven nonmelanoma skin cancer.

It is important to note that HPV-DNA tends to persist in a nonproductive state (not detectable by routine testing) with an average latency of 15-25 years. The (sexual) transmission from subclinical lesions is extremely common and the effectiveness of vaccination is accordingly reduced with the number of partners. The prophylactic vaccines on the market do not influence the natural history of HPV infection; thus, the HPV vaccine should be administrated prior to sexual exposure. Not antibodies but T-cell-mediated immunoreactions against viral oncoproteins in tumor cells mediate the spontaneous regression of intraepithelial neoplasia [5]. However, therapeutically effective vaccines in the pipeline are not yet available.

Since licensure of the HPV vaccine in 2006, vaccine coverage remains low, with the exception of ground breaking Australia. Financial concerns, misleading mar- 
keting, politically motivated erroneous claims, e.g., linking the vaccine to mental retardation or fundamentalist opposition to any vaccine are barriers to vaccination. In the United States, there is even concern about vaccine's effect on sexual behavior. Despite of excellent records, parents are unsettled about vaccine safety being unaware of the seriousness of nowadays preventable cancer! Broad and repetitive information of the public by health care professionals is urgently needed. There is the hope that the enormous benefit of this medical breakthrough that now becomes more and more obvious will increase acceptance of the HPV vaccines by the public.

\section{Conflict of interest}

Patent holder for skin test with oncoproteins E6 and E7.

\section{References}

1. Shope RE. Infectious papillomatosis of rabbits. J Exp Med. 1933;58:607-24.

2. Zur Hausen H. Q \& A.: on the case. Nature. 2012;488:16.

3. Kreider JW, et al. Morphologic transformation in vivo of human uterine cervix with papillomavirus from condylomata acuminata. Nature. 1985;317:639-41.

4. Zhou J, Sun XY, Stenzel DJ, Frazer IH. Expression of vaccinia recombinant HPV $16 \mathrm{~L} 1$ and L2 ORF protein in epithelial cells is sufficient for assembly of HPV virion-like particles. Virology. 1991;185:251-7.

5. Höpfl R, et al. Spontaneous regression of CIN and delayedtype hypersensitivity to HPV16 oncoprotein E7. The Lancet. 2000;356:1985-6. 